UCD-98-18

hep-th/9812013

November 1998

revised January 1999

\title{
Black Hole Entropy from Conformal Field Theory in Any Dimension
}

\author{
S. CARLIP \\ Department of Physics \\ University of California \\ Davis, CA 95616 \\ $U S A$
}

\begin{abstract}
Restricted to a black hole horizon, the "gauge" algebra of surface deformations in general relativity contains a Virasoro subalgebra with a calculable central charge. The fields in any quantum theory of gravity must transform accordingly, i.e., they must admit a conformal field theory description. Applying Cardy's formula for the asymptotic density of states, I use this result to derive the Bekenstein-Hawking entropy. This method is universal - it holds for any black hole, and requires no details of quantum gravity - but it is also explicitly statistical mechanical, based on counting microscopic states.
\end{abstract}

*email: carlip@dirac.ucdavis.edu 


\section{Introduction}

Since the discovery that black holes behave as thermal objects, an outstanding open question has been whether black hole thermodynamics has a "statistical mechanical" description in terms of microscopic states. An answer could shed light on broader problems of quantum gravity; at a minimum, black hole thermodynamics provides an important consistency check for any candidate theory of quantum gravity.

Until recently, we had no convincing model of microscopic black hole states. Today, we have a plethora of possibilities, from D-brane states in string theory [1] to spin network states in loop quantum gravity [2]. A fundamental issue remains, however, perhaps best described as the problem of universality [3]. The Bekenstein-Hawking entropy can be computed entirely within the framework of quantum field theory in a fixed curved background. It is hard to see how such a calculation could "know" the details of a microscopic gravitational theory. Rather, it seems more likely that some unknown universal mechanism forces any suitable quantum theory to give the standard result.

A major step toward finding a universal mechanism was taken by Strominger [4], who reanalyzed the $(2+1)$-dimensional black hole [5] using conformal field theory methods. Brown and Henneaux had shown that the asymptotic symmetry algebra for this solution was a Virasoro algebra, implying that any theory of microscopic states should be a conformal field theory [6]. Strominger observed that the Cardy formula [7] for the asymptotic growth of states could thus be used to compute the entropy, and that the result agreed with the usual Bekenstein-Hawking expression. This analysis was subsequently extended to a number of higher-dimensional black holes with near-horizon geometries resembling that of the $(2+1)$-dimensional black hole (see 8 for a partial list of references).

But while many black holes have the appropriate near-horizon geometry for such an analysis, others do not. Moreover, the Virasoro algebra of Brown and Henneaux is an algebra of asymptotic symmetries at spatial infinity, while black hole entropy should arguably be a more local property of horizons.

In this paper, I generalize Strominger's approach by looking at the symmetries of the horizon of an arbitrary black hole. The relevant algebra of surface deformations contains a physically important Virasoro algebra, essentially consisting of deformations of the $r-t$ plane that leave the horizon fixed. The analysis of Brown and Henneaux can be extended to find the central charge of this algebra. I show that the Cardy formula then yields the correct Bekenstein-Hawking entropy, independent of the details of the black hole.

\section{Metric and Boundary Terms}

Let us start with a general black-hole-like metric in $n$ spacetime dimensions,

$$
d s^{2}=-N^{2} d t^{2}+f^{2}\left(d r+N^{r} d t\right)^{2}+\sigma_{\alpha \beta}\left(d x^{\alpha}+N^{\alpha} d t\right)\left(d x^{\beta}+N^{\beta} d t\right)
$$

\footnotetext{
${ }^{*}$ Greek letters from the middle of the alphabet are spacetime indices, Roman letters are spatial indices, and Greek letters from the beginning of the alphabet are "boundary" or "angular" indices.
} 
with a lapse function $N$ that vanishes at a horizon $r=r_{+}$and behaves near $r=r_{+}$as

$$
N^{2}=h\left(x^{\alpha}\right)\left(r-r_{+}\right)+O\left(r-r_{+}\right)^{2}, \quad n^{a} \partial_{a} N=2 \pi / \beta,
$$

where $n^{a}$ is the unit normal to $r=r_{+}$on a constant $t$ slice. For a stationary black hole with coordinates such that $N^{r}=0, \beta$ is the inverse Hawking temperature, and is constant on the horizon.

I will treat $r=r_{+}$as a boundary - or, more precisely, a surface at which certain fields are fixed, and at which boundary terms are therefore needed in a variational principle [9]and will assume that the metric approaches that of a standard, momentarily stationary, black hole near this boundary. Suitable fall-off conditions near $N=0$ are

$$
\begin{aligned}
& f=\frac{\beta h}{4 \pi} N^{-1}+O(1), \quad N^{r}=O\left(N^{2}\right), \quad \sigma_{\alpha \beta}=O(1), \quad N^{\alpha}=O(1), \\
& \left(\partial_{t}-N^{r} \partial_{r}\right) g_{\mu \nu}=O(N) g_{\mu \nu}, \quad \nabla_{\alpha} N_{\beta}+\nabla_{\beta} N_{\alpha}=O(N) .
\end{aligned}
$$

The last condition is essentially the requirement that angular velocity be constant on the horizon. The extrinsic curvature of a slice of constant time then behaves as

$$
K_{r r}=O\left(1 / N^{3}\right), \quad K_{\alpha r}=O(1 / N), \quad K_{\alpha \beta}=O(1)
$$

near the horizon. (Note that $\partial_{r} N=O(1 / N)$.)

In the Hamiltonian (ADM) formulation of general relativity, the group of "gauge" symmetries is the surface deformation group, generated by the quantity

$$
H[\hat{\xi}]=\int_{\Sigma} d^{n-1} x \hat{\xi}^{\mu} \mathcal{H}_{\mu}+\text { boundary terms }
$$

where $\left\{\mathcal{H}_{t}, \mathcal{H}_{a}\right\}$ are the Hamiltonian and momentum constraints. The parameters $\hat{\xi}^{\mu}$ are almost, but not quite, identical to parameters $\xi^{\mu}$ labeling infinitesimal spacetime diffeomorphisms; the two are related by [10]

$$
\hat{\xi}^{t}=N \xi^{t}, \quad \hat{\xi}^{a}=\xi^{a}+N^{a} \xi^{t} .
$$

As usual, the variation of the "volume piece" of $H[\hat{\xi}]$ contains surface terms at the boundary - in this case, the horizon - which take the standard form [6]

$$
-\frac{1}{16 \pi G} \int_{r=r_{+}} d^{n-2} x\left\{\sqrt{\sigma}\left(\sigma^{a c} n^{b}-\sigma^{a b} n^{c}\right)\left(\hat{\xi}^{t} \nabla_{c} \delta g_{a b}-\nabla_{c} \hat{\xi}^{t} \delta g_{a b}\right)+2 \hat{\xi}^{a} \delta \pi_{a}^{r}-\hat{\xi}^{r} \pi^{a b} \delta g_{a b}\right\},
$$

where $\pi^{a b}=f \sqrt{\sigma}\left(K^{a b}-g^{a b} K\right)$ is the momentum conjugate to $g_{a b}$. To have a well-defined symmetry generator, we must add a boundary term to $H[\hat{\xi}]$ to cancel the variation (2.7). Note that the fall-off conditions (2.3)-(2.4) necessitate that

$$
\hat{\xi}^{r}=O\left(N^{2}\right), \quad \hat{\xi}^{t}=O(N), \quad \hat{\xi}^{\alpha}=O(1),
$$

since otherwise surface deformations would change the metric near $N=0$. 
It is straightforward to check that a term

$$
J[\hat{\xi}]=\frac{1}{8 \pi G} \int_{r=r_{+}} d^{n-2} x\left\{n^{a} \nabla_{a} \hat{\xi}^{t} \sqrt{\sigma}+\hat{\xi}^{a} \pi_{a}^{r}+n_{a} \hat{\xi}^{a} K \sqrt{\sigma}\right\}
$$

added to the generator (2.5) yields a variation

$$
\delta(H[\hat{\xi}]+J[\hat{\xi}])=\text { bulk terms }+\frac{1}{8 \pi G} \int_{r=r_{+}} d^{n-2} x\left(\delta n^{r} \partial_{r} \hat{\xi}^{t}+\frac{1}{f} \hat{\xi}^{r} \delta K_{r r}+\delta n_{r} \hat{\xi}^{r} K\right) \sqrt{\sigma}
$$

In constrast to more familiar variational problems, the normal $n^{a}$ need not be fixed at the boundary, but $\delta n^{r}$ can be computed from the requirement that $\delta\left(g_{a b} n^{a} n^{b}\right)=0$. If we now restrict our variations to those satisfying $\delta f / f=O(N)$ and $\delta K_{r r} / K_{r r}=O(N)$, the boundary term in (2.10) vanishes, as required.

A useful check of eqn. (2.9) can be obtained by specializing to variations $\delta H$ that are themselves surface deformations. Let $L[\hat{\xi}]=H[\hat{\xi}]+J[\hat{\xi}]$ be the full generator of surface deformations. Then the deformation of $L[\hat{\xi}]$ should itself be generated by $L[\hat{\xi}]$ : that is, it should be given by the Poisson bracket [6]

$$
\delta_{\hat{\xi}_{2}} L\left[\hat{\xi}_{1}\right]=\left\{L\left[\hat{\xi}_{2}\right], L\left[\hat{\xi}_{1}\right]\right\}=L\left[\left\{\hat{\xi}_{1}, \hat{\xi}_{2}\right\}_{\mathrm{SD}}\right]+K\left[\hat{\xi}_{1}, \hat{\xi}_{2}\right]
$$

where $K\left[\hat{\xi}_{1}, \hat{\xi}_{2}\right]$ is a possible central term in the algebra. Here $\left\{\hat{\xi}_{1}, \hat{\xi}_{2}\right\}_{\mathrm{SD}}$ is the Lie bracket for the algebra of surface deformations, given by [10]

$$
\begin{aligned}
& \left\{\hat{\xi}_{1}, \hat{\xi}_{2}\right\}_{\mathrm{SD}}^{t}=\hat{\xi}_{1}^{a} \partial_{a} \hat{\xi}_{2}^{t}-\hat{\xi}_{2}^{a} \partial_{a} \hat{\xi}_{1}^{t} \\
& \left\{\hat{\xi}_{1}, \hat{\xi}_{2}\right\}_{\mathrm{SD}}^{a}=\hat{\xi}_{1}^{b} \partial_{b} \hat{\xi}_{2}^{a}-\hat{\xi}_{2}^{b} \partial_{b} \hat{\xi}_{1}^{a}+g^{a b}\left(\hat{\xi}_{1}^{t} \partial_{b} \hat{\xi}_{2}^{t}-\hat{\xi}_{2}^{t} \partial_{b} \hat{\xi}_{1}^{t}\right) .
\end{aligned}
$$

The equality (2.11) will be used in the next section to compute the central charge. For now, let us note that when evaluated at a stationary black hole metric in standard coordinates, for which $K_{r r}=0=K_{\alpha \beta}$, the boundary term in (2.10) becomes

$$
-\frac{1}{8 \pi G} \int_{r=r_{+}} d^{n-2} x \sqrt{\sigma}\left\{\frac{1}{f^{2}}\left[\partial_{r}\left(f \hat{\xi}_{2}^{r}\right) \partial_{r} \hat{\xi}_{1}^{t}-\partial_{r}\left(f \hat{\xi}_{1}^{r}\right) \partial_{r} \hat{\xi}_{2}^{t}\right]+\frac{1}{f} \partial_{r}\left[\hat{\xi}_{1}^{r} \partial_{r} \hat{\xi}_{2}^{t}-\delta_{\hat{\xi}_{2}} \hat{\xi}_{1}^{t}\right]\right\} .
$$

If we assume, as suggested by eqn. (2.12), that $\delta_{\hat{\xi}_{2}} \hat{\xi}_{1}^{t}=\hat{\xi}_{2}^{a} \partial_{a} \hat{\xi}_{1}^{t}$, then this expression is antisymmetric in $\hat{\xi}_{1}$ and $\hat{\xi}_{2}$, as required by eqn. (2.11). Our boundary terms are thus consistent with the interpretation of $L[\hat{\xi}]$ as the generator of surface deformations in the presence of a horizon.

\section{The Virasoro Algebra}

In the preceding section, we considered general variations of a general black-hole-like metric. Let us now specialize to the case of an axially symmetric black hole, with an 
adapted angular coordinate $\phi$ such that $\partial_{\phi} g_{\mu \nu}=0$. For simplicity, I will assume that only the component $N^{\phi}$ of the shift vector is nonzero; the higher-dimensional generalization to more than one rotational Killing vector is straightforward.

We now focus our attention on a particular subalgebra of the surface deformation algebra with the following three properties:

1. The surface deformations are restricted to the $r-t$ plane. This specialization is inspired by the path integral approach to black hole thermodynamics, in which it is clear that the $r-t$ plane has the central role in determining the entropy [11].

2. The diffeomorphism parameter $\xi^{t}=\hat{\xi}^{t} / N$ "lives on the horizon," in the sense that near $r=r_{+}$it depends on $t$ and $r$ only in the combination $t-r_{*}$, where $f d r=N d r_{*}$ in the time-slicing such that $N_{r}=0$. For the Kerr black hole, $t-r_{*}$ is essentially the standard Eddington-Finkelstein retarded time, up to corrections of order $r-r_{+}$.

3. The lapse function $N^{2}$ is fixed at the horizon. The horizon is physically defined by the condition $N^{2}=0$, while our boundary term (2.9) is written at $r=r_{+}$; this condition ensures that the boundary remains at the horizon.

Condition 1 and eqn. (2.6) imply that the diffeomorphism parameter $\xi^{\phi}$ has the form

$$
\xi^{\phi}=-N^{\phi} \xi^{t}=-\frac{N^{\phi}}{N} \hat{\xi}^{t}
$$

Condition 2 requires that

$$
\partial_{r} \xi^{t}=-\frac{f}{N} \partial_{t} \xi^{t}
$$

at $r=r_{+}$, allowing us to write radial derivatives at the horizon in terms of time derivatives. To impose condition 3, we can examine diffeomorphisms of $g^{t t}=-1 / N^{2}$. With initial coordinates chosen so that $N_{r}=0$, we find that

$$
\delta g^{t t}=0=\frac{2}{N^{2}}\left(\partial_{t}-N^{\phi} \partial_{\phi}\right) \xi^{t}+\frac{h}{N^{4}} \xi^{r}
$$

This structure suggests that we split our diffeomorphisms into left-moving modes $\xi^{t}$, for which $\partial_{t} \xi^{t}=\Omega \partial_{\phi} \xi^{t}$, and right-moving modes $\tilde{\xi}^{t}$, for which $\partial_{t} \tilde{\xi}^{t}=-\Omega \partial_{\phi} \tilde{\xi}^{t}$, where $\Omega=-N^{\phi}\left(r_{+}\right)$is the angular velocity of the horizon. Then

$$
\xi^{r}=-\frac{4 N^{2}}{h} \partial_{t} \xi^{t}, \quad \tilde{\xi}^{r}=0
$$

Note that $\xi^{r_{*}}=(f / N) \xi^{r}$ is, like $\xi^{t}$, a function of retarded time $t-r_{*}$ at the horizon.

We can use these results to write the left-moving modes at the horizon as

$$
\xi_{n}^{t}=\frac{T}{4 \pi} \exp \left\{\frac{2 \pi i n}{T}\left(t-r_{*}+\Omega^{-1} \phi\right)\right\},
$$


where $T$ is an arbitrary period. (A possible choice is $T=\beta$, which matches the periodicity of the Euclidean black hole, but as we shall see, $T$ drops out of the final expression for the entropy.) The normalization (3.5) has been fixed by the requirement that the surface deformation algebra (2.12) reproduce the Diff $S^{1}$ algebra

$$
\left\{\hat{\xi}_{m}, \hat{\xi}_{n}\right\}_{\mathrm{SD}}^{t}=i(n-m) \hat{\xi}_{m+n}^{t} .
$$

Substituting the modes (3.5) into the boundary term (2.13), we obtain

$$
\delta_{\hat{\xi}_{m}} L\left[\hat{\xi}_{n}\right]=\text { bulk terms }+\frac{A}{8 \pi G} \frac{\beta}{T} i n^{3} \delta_{m+n},
$$

where $A$ is the area of the boundary at $r=r_{+}$. We can now use a trick of Brown and Henneaux to evaluate the central term $K\left[\hat{\xi}_{m}, \hat{\xi}_{n}\right]$. When evaluated on shell, the Hamiltonian and momentum constraints vanish, so $H[\hat{\xi}]=0$. Equation (2.11) thus reduces to a collection of boundary terms,

$$
\frac{A}{8 \pi G} \frac{\beta}{T} i n^{3} \delta_{m+n}=J\left[\left\{\hat{\xi}_{m}, \hat{\xi}_{n}\right\}_{\mathrm{SD}}\right]+K\left[\hat{\xi}_{m}, \hat{\xi}_{n}\right]=i(n-m) J\left[\hat{\xi}_{m+n}\right]+K\left[\hat{\xi}_{m}, \hat{\xi}_{n}\right]
$$

From eqn. (2.9), it is easily checked that

$$
J\left[\hat{\xi}_{p}\right]=\frac{A}{16 \pi G} \frac{T}{\beta} \delta_{p 0}
$$

on shell. Hence

$$
K\left[\hat{\xi}_{m}, \hat{\xi}_{n}\right]=\frac{A}{8 \pi G} \frac{\beta}{T} i n\left(n^{2}-\frac{T^{2}}{\beta^{2}}\right) \delta_{m+n},
$$

the correct formin for the central term of a Virasoro algebra with central charge

$$
c=\frac{3 A}{2 \pi G} \frac{\beta}{T} .
$$

\section{Counting States}

The models we are investigating are not two-dimensional. Nevertheless, the results above imply that the quantum states that characterize a black hole horizon must transform under a Virasoro algebra with central charge (3.11). This is sufficient to permit the use of powerful methods from conformal field theory to count states.

In particular, a conformal field theory with a central charge $c$ has a density of states $\rho\left(L_{0}\right)$ that grows asymptotically as

$$
\log \rho\left(L_{0}\right) \sim 2 \pi \sqrt{\frac{c_{\mathrm{eff}} L_{0}}{6}},
$$

\footnotetext{
${ }^{\dagger}$ The $n$ dependence in $(3.10)$ can be made the usual $n\left(n^{2}-1\right)$ by shifting $L_{0}$ by a constant. This alters the eigenvalue (3.9), but also changes the "effective central charge" so that the entropy (4.2) is unaffected.
} 
where $c_{\text {eff }}$ is an "effective central charge" [7, 12]. If the spectrum satisfies reasonable, although not universal, conditions [8]-notably that the ground state is an eigenstate of $L_{0}$ with eigenvalue zero - then $c_{\mathrm{eff}}=c$. Following Strominger [4], let us assume these conditions are satisfied in quantum gravity. Then from eqns. (3.9) and (3.11),

$$
\log \rho\left(L_{0}\right) \sim \frac{A}{4 G}
$$

recovering the standard Bekenstein-Hawking entropy. In general, right-moving modes may make an additional contribution to the density of states, but it is clear from eqn. (3.4) that the central charge for those modes vanishes, so eqn. (4.2) gives the full entropy.

\section{Four Questions and Two Answers}

The analysis above strongly suggests that any quantum description of black hole horizon states must yield the standard Bekenstein-Hawking entropy. Here, I will briefly address some details of this analysis and raise several remaining questions.

1. What is the significance of the boundary condition $N=0$ ?

For a stationary black hole, in the coordinates $N^{r}=0, f \sim 1 / N$ that we used to evaluate the central charge, this is the condition for an apparent horizon. In other coordinates, however, the apparent horizon condition is considerably more complicated. An investigation of possible alternative boundary conditions might help answer the question of what kind of "horizon" is needed for black hole entropy. It may also be possible to extend this analysis to more general gravitational actions along the lines of Ref. [13].

2. The Cardy formula (4.1) comes from two-dimensional conformal field theory. What are the two relevant dimensions here?

The Cardy formula requires a modular invariant partition function of the form

$$
Z=\operatorname{Tr} \exp \{i(J \phi+E t)\}
$$

where $J$ and $E$ are conserved charges associated with translations in $\phi$ and $t$. For an axially symmetric black hole, $\phi$ and $t$ are determined by the two Killing vectors, and modular invariance is a consequence of diffeomorphism invariance. The two "preferred" directions are thus picked out by the symmetries. (For a black hole in more than four dimensions with more than one axial Killing vector, the left-moving modes are determined by the condition $\partial_{t} \xi^{t}=-N^{\alpha} \partial_{\alpha} \xi^{t}$, so the shift vector $N^{\alpha}$ picks out an angular direction.)

3. What specific degrees of freedom account for the entropy (4.2)?

Like Strominger's derivation [4], this computation does not address this question, but rather uses symmetry arguments to derive the behavior of any microscopic theory of 
black hole horizon states. This is both good and bad: good because it provides a universal explanation of black hole statistical mechanics, bad because it offers little further insight into quantum gravity.

One possible picture of the microscopic degrees of freedom comes from considering the dimensional reduction of Einstein gravity to the $r-t$ plane near a horizon. The resulting action contains a scalar field, essentially $\sqrt{\sigma}$, that couples to the two-dimensional scalar curvature ${ }^{(2)} R$. The action is not conformally invariant, but we know from the $c$ theorem [14] that it must flow to a conformal field theory - presumably a Liouville theory - under the renormalization group. Since the dimensionally reduced action has a prefactor of $A / 16 \pi G$, the central charge of such a Liouville theory is likely to be proportional to $A / 16 \pi G$, and might reproduce the central charge (3.11). De Alwis has considered a similar renormalization group flow in a somewhat different context [15], and Solodukhin has recently proposed a related analysis of dimensionally reduced gravity [16].

It also seems plausible that the description of black hole entropy here is related to the picture of microscopic states as "would-be pure gauge" degrees of freedom that become dynamical at a boundary [9, 17]. The existence of a central charge is an indication that the algebra of surface deformations has become anomalous, and that invariance cannot be consistently imposed on all states. This connection has not been developed, however.

4. Does the relevant conformal field theory satisfy the technical conditions required for the Cardy formula? In particular, does the ground state have $L_{0}=0$ ?

Without a much more detailed description of the conformal field theory, this question cannot be answered. My approach differs from Strominger's in an important respect. Strominger's boundary conditions were those of anti-de Sitter space, offering the possibility that anti-de Sitter space is the ground state. The boundary conditions of this paper are those of a specific black hole, and depend on the horizon metric. This difference is hard to avoid, since without the extra length scale provided by a cosmological constant it is difficult to write down a dimensionless central charge independent of the boundary values of the metric. (One could, of course, choose $T$ to be proportional to $A / \beta$ in eqn. (3.11), but there seems to be little physical justification for such a choice.)

There is, however, a plausible candidate for the ground state in the model developed here: the extremal black hole, which is typically characterized by a lapse function behaving as $N^{2} \sim\left(r-r_{+}\right)^{2}$ near the horizon. Such a configuration satisfies the boundary conditions assumed in this paper, but in contrast to the nonextremal result (3.9), eqn. (2.9) now gives $J\left[\hat{\xi}_{0}\right]=0$, implying that at least the classical contribution to $L_{0}$ vanishes.

\section{Acknowledgements}

I would like to thank Rob Meyers for pointing out some errors in the first version of this paper. This work was supported in part by National Science Foundation grant PHY-93-57203 and Department of Energy grant DE-FG03-91ER40674. 


\section{References}

[1] See, for example, A. Strominger and C. Vafa, Phys. Lett. B379, 99 (1996).

[2] See, for example, A. Ashtekar et al., Phys. Rev. Lett. 80, 904 (1998).

[3] A. Strominger, talk at the Spinoza Meeting on the Quantum Black Hole, Utrecht, 1998.

[4] A. Strominger, J. High Energy Phys. 02, 009 (1998).

[5] M. Bañados, C. Teitelboim, and J. Zanelli, Phys. Rev. Lett. 69, 1849 (1992).

[6] J. D. Brown and M. Henneaux, Commun. Math. Phys. 104, 207 (1986).

[7] J. A. Cardy, Nucl. Phys. B270, 186 (1986); H. W. J. Blöte, J. A. Cardy, and M. P. Nightingale, Phys. Rev. Lett. 56, 742 (1986).

[8] S. Carlip, Class. Quantum Grav. 15, 3609 (1998).

[9] S. Carlip, in Contrained Dynamics and Quantum Gravity 1996, edited by V. de Alfaro et al., Nucl. Phys. B (Proc. Suppl.) 57, 8 (1997).

[10] J. D. Brown, Lower Dimensional Gravity (World Scientific, Singapore, 1988).

[11] M. Bañados, C. Teitelboim, and J. Zanelli, Phys. Rev. Lett. 72, 957 (1994).

[12] D. Kutasov and N. Seiberg, Nucl. Phys. B358, 600 (1991).

[13] J. D. Brown, Phys. Rev. D52, 7011 (1995).

[14] A. B. Zamolodchikov, JETP Lett. 43, 730 (1986); Sov. J. Nucl. Phys. 46, 1090 (1987).

[15] S. P. de Alwis, Phys. Rev. D46, 5429 (1992).

[16] S. N. Solodukhin, Utrecht preprint SPIN-1998/10, e-print hep-th/9812056.

[17] A. P. Balachandran, L. Chandar, and A. Momen, Nucl. Phys. B461, 581 (1996). 